\title{
OBSERVATION OF OPTICAL BISTABILITY IN CHOLESTERIC LIQUID CRYSTALS
}

\author{
B. Pura, I. Gronowska and J. Petykiewicz \\ Institute of Physics, Warsaw University of Technology \\ Koszykowa 75, 00-662 Warszawa, Poland \\ (Received January 21, 1997; revised version May 15, 1997) \\ In the paper, some experimental and theoretical results concerning op- \\ tical bistability in cholesteric liquid crystals are presented. A strong primary \\ laser beam interacted nonlinearly with the material and influenced a second \\ (probe) beam in such a way that its transmitted part exhibited a bistable \\ dependence on the intensity of the first beam. It was observed that such a \\ character may be attributed to the intensity of the outgoing probe wave. \\ The obtained results reflect the symmetry of the sample and are interpreted \\ as second order nonlinearity effects.
}

PACS numbers: 42.65.Pc, 42.70.-a

\section{Introduction}

Cholestcric liquid crystals (CLCs) possess an intrinsic helical structure which leads to some unusual optical properties in the linear regime. This structure influences significantly both the intensity as well as the polarization of the wave propagating through such a crystal. Recent investigations of the optical properties of CLCs concentrate rather on their nonlinear effects, among them on the optical bistability.

Optical bistability still belongs to the most important optical nonlinear effects $[1,2]$. The basic mechanisms of bistability and the means of observing it are numerous. Most of the optical bistable devices are manufactured with the aid of the Fabry-Perot cavity, in which the nonlinear material is located. The power of the optical field required to achieve bistability varied in a wide range, which depends on the nonlinearity of the material used.

In this paper we report the observation of optical bistability created by a cholesteric liquid crystal film. A strong primary laser beam of a polarized light changed the electric polarization of the medium which, in turn, changed the conditions for the bistability to appear. Such reoriented sample was then exposed to a second laser beam of a much smaller intensity (called the probe beam) which 
was partially transmitted. The behaviour of the second (outgoing) wave depended essentially on the geometry of the experimental setup and on the parameters of the first (stronger) wave, i.e., on its intensity and polarization. Under some conditions there appeared the bistability of intensity of the probe beam. A new model of these phenomena in the medium with second-order nonlinearity is the main theoretical aim of this paper.

\section{Experiment}

The experimental setup is shown in Fig. 1. The bean indicated by (1) came from the argon-ion laser (LEXEL 3500) operating at the $5145 \AA$ (green). Its power $P_{1}$ was varied from $0.05 \mathrm{~W}$ to $1.2 \mathrm{~W}$. Additionally, by means of a linear polarizer we changed the polarization angle $\theta$ of the first beam in the range of $0^{\circ}-90^{\circ}$. The angle $0^{\circ}$ corresponded approximately to the vertical direction, parallel to the surface of the CLC film considered. First measurements were performed without the use of the polarizer, but even then the beam was partially polarized by the laser itself. The intensity of the beam from the helium-neon laser was kept constant. On the other hand, the intensity of the green (strong primary) wave was changed in a wide range.

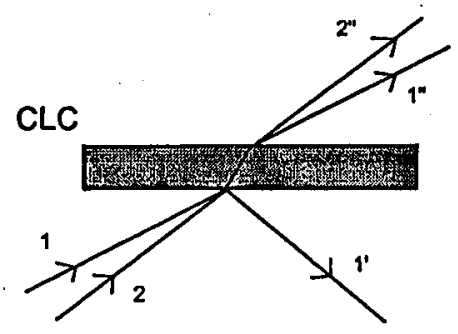

Fig. 1. The experimental setup.

The probe beam (2) came from the second (helium) laser of the power of the order $40 \mathrm{~mW}$, operating at $\lambda=6320 \AA$ (red). It fell at the same region as the first (green) wave. Interaction of both waves, via the medium, led to the significant changes of the intensity of the outgoing wave $2^{\prime \prime}$ denoted further by $P_{2}$.

The liquid crystal film was oriented by means of the dc electric field of the voltage $1.5 \mathrm{~V}$, perpendicular to the film of the thickness $30 \mu \mathrm{m}$. Due to this field the molecules of the CLC had in general similar directions.

\section{Results}

The transmitted red light was a very sensitive function of the intensity of the original green beam. This dependence is presented in Fig. 2.

We observed that $P_{2}$ grew abruptly with increasing $P_{1}$ at $P_{1} \approx 0.6 \mathrm{~W}$ (see the lower curve). By decreasing $P_{1}$ a similar jump (this time down) appeared at $P_{1} \approx 0.5 \mathrm{~W}$ (see the upper curve). That means that the width of the bistable loop was about $0.1 \mathrm{~W}$. More spectacular was the height of this loop. In all the cases it reached more than 3 times of the initial value. 


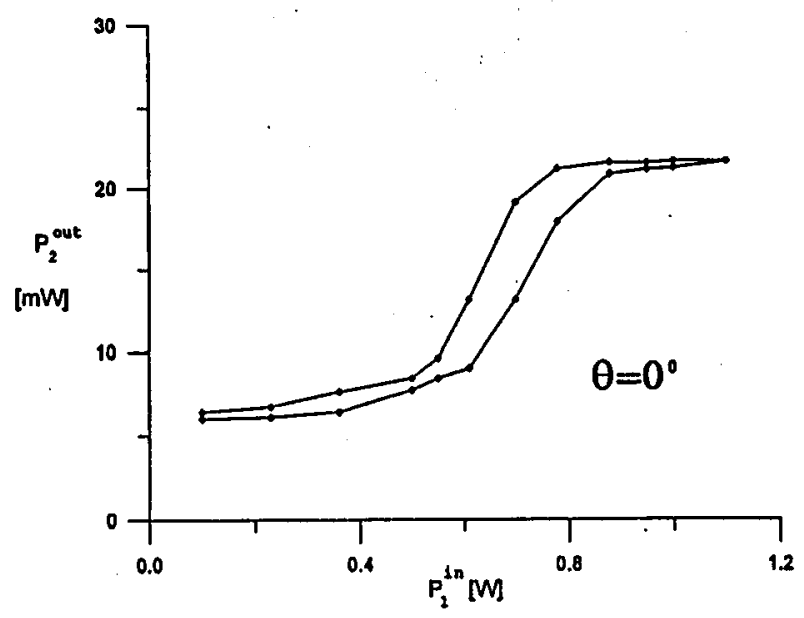

Fig. 2. Typical plot of the bistabile loop for the transmitted probe beam as a function of the intensity of incoming strong laser beam.

The polarization of the green light had significant influence on the shape of all curves. The maximal intensity of outgoing red beam became smaller with increasing angle of polarization. For $\Theta>60^{\circ}$ the bistable loop practically disappeared.

\section{Theoretical explanation \\ 4.1. General remarks}

Let assume that two different plane monochromatic waves with frequencies $\omega_{1}$ and $\omega_{2}\left(\omega_{1}>\omega_{2}\right)$ and wave vectors $k_{1}$ and $k_{2}$ fall at the liquid crystal film.

The second order nonlinear polarization has the following form [3]:

$P^{\mathrm{NL}}=\epsilon_{0} \overline{\bar{\chi}}: \boldsymbol{E} \cdot \boldsymbol{E}$,

where $\overline{\bar{\chi}}$ is the third order electric nonlinear permeability tensor of the CLC, $\epsilon_{0}$ is the vacuum permeability.

The resultant primary electric field in Eq. (1) is composed of the two monochromatic planary waves with frequencies $\omega_{1}$ and $\omega_{2}$

$$
\boldsymbol{E}_{\mathrm{I}}=\boldsymbol{E}\left(\omega_{1}\right)+\boldsymbol{E}\left(\omega_{2}\right) \text {. }
$$

These two monochromatic waves induce in the medium the nonlinear polarization with the following secondary frequencies: $\omega_{\mathrm{I}}=\omega_{1} \pm \omega_{2}, \omega_{\mathrm{II}}=2 \omega_{1}, \omega_{\mathrm{III}}=$ $2 \omega_{2}$ and the static field $\omega_{\text {IV }}=0$.

The secondary monochromatic waves with frequencies $\omega_{3}=\omega_{1} \pm \omega_{2}$ have the wave vector $k_{3}$ for which

$$
\Delta k=k_{1}-\left(k_{2}+k_{3}\right) \neq 0 \text {. }
$$

The existence of the phase mismatch $(\Delta k \neq 0)$ results in an insignificant increase in the $a_{3}(z)$ amplitude of the secondary monochromatic wave. If $a_{3}(0)$ is very small compared to the $a_{1}(0)$ amplitude of the strong $\omega_{1}$ wave at $z=0$, 
it remains small for all values of $z$ (the $z$-axis indicates the wave propagation direction).

Because of the condition (3), i.e., the presence of the phase mismatch, the static component of the nonlinear polarization of the medium has a significant influence on the transmission properties through the CLC liquid crystal film, for the waves with primary frequencies. The static component has the following form:

$$
\boldsymbol{P}^{\mathrm{NL}}\left(\omega_{\mathrm{IV}}=0\right)=\epsilon_{0} \overline{\overline{\bar{\chi}}}:\left[\boldsymbol{E}\left(\omega_{1}\right) \cdot \boldsymbol{E}^{*}\left(\omega_{1}\right)+\boldsymbol{E}\left(\omega_{2}\right) \cdot \boldsymbol{E}^{*}\left(\omega_{2}\right)\right],
$$

where $*$ denotes the complex conjugation.

This component of the nonlinear polarization generates the static electric field inside the considered medium

$$
\boldsymbol{E}\left(\omega_{\mathrm{IV}}=0\right)=\epsilon_{0}^{-1} \boldsymbol{P}^{\mathrm{NL}}\left(\omega_{\mathrm{IV}}=0\right),
$$

where $\epsilon_{0}^{-1}$ is the inverse of the vaccum permeability.

\subsection{Effect of cascaded nonlinearily}

The static electric field and the primary monochromatic waves produce the resultant electric field inside the considered medium in the following form:

$$
\boldsymbol{E}_{\mathrm{II}}=\boldsymbol{E}\left(\omega_{\mathrm{IV}}=0\right)+\boldsymbol{E}\left(\omega_{1}\right)+\boldsymbol{E}\left(\omega_{2}\right) \text {. }
$$

This field after utilizing Eq. (1) (this is the so-called cascaded bistability effect [4]) generates among others secondary components of the nonlinear polarization of the medium with frequencies $\omega_{1}$ and $\omega_{2}$ :

$$
\begin{aligned}
& P^{\mathrm{NL}}\left(\omega_{1}\right)=\epsilon_{0} \overline{\overline{\bar{\chi}}}: \boldsymbol{E}\left(\omega_{\mathrm{IV}}=0\right) \boldsymbol{E}\left(\omega_{1}\right), \\
& \boldsymbol{P}^{\mathrm{NL}}\left(\omega_{2}\right)=\epsilon_{0} \overline{\overline{\bar{\chi}}}: \boldsymbol{E}\left(\omega_{\mathrm{IV}}=0\right) \boldsymbol{E}\left(\omega_{2}\right) .
\end{aligned}
$$

Because of these cascaded nonlinearities, the primary fields with frequencies $\omega_{1}$ and $\omega_{2}$ should fulfil the following equation (see [5]):

$$
\operatorname{curlcurl} \boldsymbol{E}\left(\omega_{j}\right)-\mu_{0} \overline{\bar{\epsilon}}_{L} \omega_{j}^{2} \boldsymbol{E}\left(\omega_{j}\right)=\mu_{0} \omega_{j}^{2} \boldsymbol{P}^{\mathrm{NL}}\left(\omega_{j}\right) \text {. }
$$

\subsection{Equations of wave motion}

Because of the finite thickness of the CLC film considered $(0 \leq z \leq L)$, there appears the reflection of waves from the boundary planes of the film. The presence of wave reflections and nonlinear polarizations (7) require the following representation of each of the monochromatic waves inside the CLC film:

$$
\boldsymbol{E}\left(\omega_{j}\right)=\boldsymbol{e}_{j} A_{j}(z) \exp \left(-\mathrm{i} k_{j} z+\mathrm{i} \omega_{j} t\right)+\boldsymbol{e}_{j}^{\prime} B_{j}(z) \exp \left(+\mathrm{i} k_{j} z++\mathrm{i} \omega_{j} t\right),
$$

where $e_{j}$ and $\boldsymbol{e}_{j}^{\prime}$ are the polarization versors of the "forward" and the "backward" propagating waves, respectively. In the following we will assume $e_{j}=e_{j}^{\prime} . A_{j}(z)$ and $B_{j}(z)$ are their respective complex amplitudes, i.e.

$$
\begin{aligned}
& A_{j}(z)=a_{j}(z) \exp \left(\mathrm{i} \psi_{j}(z)\right) \\
& B_{j}(z)=b_{j}(z) \exp \left(\mathrm{i} \phi_{j}(z)\right),
\end{aligned}
$$

where $a_{j}(z), b_{j}(z)$ and $\psi_{j}(z), \phi_{j}(z)$ are real amplitudes and phases, respectively [6]. 
From Eqs. (8), (5) and Eq. (7) it follows that the equations governing the motion of the "forward" propagating waves are

$$
\begin{aligned}
& \frac{\mathrm{d} A_{1}}{\mathrm{~d} z}=\mathrm{i}\left[\gamma_{11}\left(A_{1} A_{1}^{*}+B_{1} B_{1}^{*}\right)+\gamma_{12}\left(A_{2} A_{2}^{*}+B_{2} B_{2}^{*}\right)\right] A_{1}, \\
& \frac{\mathrm{d} A_{2}}{\mathrm{~d} z}=\mathrm{i}\left[\gamma_{21}\left(A_{1} A_{1}^{*}+B_{1} B_{1}^{*}\right)+\gamma_{22}\left(A_{2} A_{2}^{*}+B_{2} B_{2}^{*}\right)\right] A_{2},
\end{aligned}
$$

where

$$
\gamma_{i j}=\frac{1}{2 n_{j} \omega_{j} c} e_{i} \overline{\overline{\bar{\chi}}}\left(\overline{\overline{\bar{\chi}}}: e_{j} e_{j}\right) e_{i}, \quad i, j=1,2,
$$

$n_{j}$ is the linear refractive index, $\omega_{j}$ is the wave frequency.

For "backward" propagating waves the respective equations are as follows:

$$
\begin{aligned}
& \frac{\mathrm{d} B_{1}}{\mathrm{~d} z}=-\mathrm{i}\left[\gamma_{11}\left(A_{1} A_{1}^{*}+B_{1} B_{1}^{*}\right)+\gamma_{12}\left(A_{2} A_{2}^{*}+B_{2} B_{2}^{*}\right)\right] B_{1}, \\
& \frac{\mathrm{d} B_{2}}{\mathrm{~d} z}=-\mathrm{i}\left[\gamma_{21}\left(A_{1} A_{1}^{*}+B_{1} B_{1}^{*}\right)+\gamma_{22}\left(A_{2} A_{2}^{*}+B_{2} B_{2}^{*}\right)\right] B_{2} .
\end{aligned}
$$

From Eqs. (11) and (13), assuming that the considered medium is transparent (i.e., $\overline{\bar{\epsilon}}_{\mathrm{L}}$ and $\overline{\overline{\bar{\chi}}}$ tensors are real), one obtains the result that the real amplitudes of the counter-propagating waves $a_{j}(z)$ and $b_{j}(z)(j=1,2)$ remain constant, independent of the variable $z$. The respective phases of these waves obey the following equations:

$$
\begin{aligned}
& \frac{\mathrm{d} \psi_{1}}{\mathrm{~d} z}=\left[\gamma_{11}\left(a_{1}^{2}+b_{1}^{2}\right)+\gamma_{12}\left(a_{2}^{2}+b_{2}^{2}\right)\right], \\
& \frac{\mathrm{d} \psi_{2}}{\mathrm{~d} z}=\left[\gamma_{21}\left(a_{1}^{2}+b_{1}^{2}\right)+\gamma_{22}\left(a_{2}^{2}+b_{2}^{2}\right)\right] ; \\
& \frac{\mathrm{d} \phi_{1}}{\mathrm{~d} z}=-\left[\gamma_{11}\left(a_{1}^{2}+b_{1}^{2}\right)+\gamma_{12}\left(a_{2}^{2}+b_{2}^{2}\right)\right], \\
& \frac{\mathrm{d} \phi_{2}}{\mathrm{~d} z}=-\left[\gamma_{21}\left(a_{1}^{2}+b_{1}^{2}\right)+\gamma_{22}\left(a_{2}^{2}+b_{2}^{2}\right)\right] .
\end{aligned}
$$

From these equations, utilizing the constancy of the real amplitudes $a_{j}$ and $b_{j}$, we obtain the following equations for the pliases of the counter-propagating waves:

$$
\begin{aligned}
& \psi_{j}(z)=+\Gamma_{j} z+\psi_{j}(0), \\
& \phi_{j}(z)=-\Gamma_{j} z+\phi_{j}(0),
\end{aligned}
$$

where

$$
\Gamma_{j}=\left[\gamma_{j 1}\left(a_{1}^{2}+b_{1}^{2}\right)+\gamma_{j 2}\left(a_{2}^{2}+b_{2}^{2}\right)\right], \quad j=1,2 .
$$

\subsection{Boundary conditions and transmitted intensities}

The counter-propagating waves are mutually connected through the boundary conditions at the boundary planes of the CLC film.

Utilizing the amplitude coefficients of reflection $r$ and transmission $\tau$ the following equations may be written: 
for $z=0$

$$
\begin{array}{ll} 
& a_{j}(0) \tau+r b_{j}(0) \exp \left(\mathrm{i} \phi_{j}(0)+\mathrm{i} k_{j} L\right)=a_{j}(0) \exp \left(\mathrm{i} \psi_{j}(0)\right), \\
\text { for } \quad z=L & \\
& b_{j}(L) \exp \left(\mathrm{i} \phi_{j}(0)\right)=r a_{j}(L) \exp \left(\mathrm{i} \psi_{j}(L)-\mathrm{i} k_{j} L\right), \quad j=1,2,
\end{array}
$$

where $a_{j 0}$ is the amplitude of the incident wave with frequency $\omega_{j}$ falling from the outside of the CLC.

If we utilize the constancy of the real amplitudes of the waves

$$
\begin{aligned}
& a_{j}(0)=a_{j}(L), \\
& b_{j}(0)=b_{j}(L)=r a_{j}(L),
\end{aligned}
$$

we obtain from Eqs. (18) the following expression for the real amplitude of the $j$-th wave inside the CLC:

$$
a_{j}(L)=\frac{a_{j 0} \tau}{\sqrt{\left(1-r^{2}\right)^{2}+4 r^{2} \sin ^{2}\left(\psi_{j}(L)-\psi_{j}(0)-k_{j} L\right) / 2}} .
$$

The light intensity of the $j$-th wave transmitted through the CLC is as follows:

$$
I_{j}^{\mathrm{tr}} \approx\left|a_{j}^{\mathrm{tr}}\right|^{2}=\left|a_{j}(L)\right|^{2}|\tau|^{2} \text {. }
$$

Taking into account Eqs. (16), (17), (20) and (21) we obtain the following expression for the intensities transmitted through the CLC film:

$$
I_{j}^{\mathrm{tr}}=\frac{|\tau|^{4} I_{0 j}}{\left(1-r^{2}\right)^{2}+4 r^{2} \sin ^{2}\left(\frac{1+r^{2}}{2 \tau^{2}}\left(\gamma_{j 1} I_{1}^{\mathrm{tr}}+\gamma_{j 2} I_{2}^{\mathrm{tr}}\right)\right)}, j=1,2
$$

where $I_{j 0}$ is the intensity of the incident wave.

The expression (22) is a typical relation describing the bistability effect in the nonlinear Fabry-Perot cavity. It not only indicates the possibility of the bistable dependence of the intensity of the wave transmmitted through the CLC film on the intensity of the incident wave, but also explains the way in which the stronger monochromatic wave can influence the transmitted intensity of the second probe wave.

Two relations (22) for $j=1,2$ are mutually conjugate. Each of the transmitted intensities depend on both primary intensities incident on the CLC film. The graphical analysis of these relations requires numerical calculations, these will be presented in a separate publication.

We would note here also the dependence of the $\gamma_{i j}$ parameters (12) on the polarization versors $e_{j}$ of the primary interacting waves. This dependence explains the influence of the polarization of these primary waves on the intensities transmitted through the CLC film. Such a dependence was indeed determined experimentally (see Sect. 2 of this paper).

\section{Discussion}

The theory of effects described above is still unsatisfactory. There exist numerous papers on this subject $([7,8]$ and references therein), in which, however, only third order nonlinearity is taken into account. In our experiment we had two 
beams going almost parallel, so that they may be treated as one composed wave which, in the first approximation, may be put in the formula derived for one wave. The total incident wave is effectively a variable of the green light only. The total outgoing wave consisted of two ones, but we extracted one of them which was relatively much more sensitive to changes in the molecular network of the CLC film.

The observed behaviour may be formally attributed to the properties of the nonlinear susceptibility tensor $\overline{\bar{\chi}}$. Cholesteric liquid crystals belong to non-centrosymmetric materials in which second order effects appear. A rough estimation (to be published elsewhere) showed that such type of optical bistability may occur in real samples provided that the polarization state of both incident waves is properly chosen.

Physical background of the obscrved effects is more complicated. A typical approach takes into account changes in molecular orientation. By minimizing the total free energy (including deformations of the crystal and the energy of the wave) one obtains nonlinear equations for the electric field $\boldsymbol{E}$ of the propagating wave (see $[2,9]$ and references therein for more details), which can be solved numerically giving the typical bistable loop.

Another class of mechanisms responsible for the optical bistability is connected with changes of the temperature [10]. The temperature increase may change the refractive index $n$, which is often used in analyzing nonlinear optical effects. In our experiments thermal effects. seemed to be of insignificant importance because the sample (very thin) was kept in between two thick plates which stabilized the temperature.

\section{Acknowledgments}

This work was supported by the grant No. 8 S507 01806 of the Committee for Scientific Research,

\section{References}

[1] H.M. Gibbs, Oplical Bistability, Academic Press, New York 1985.

[2] I.C. Khoo, Nonlinear Optics of Liquid Crystals, in series Progress in Optics, Vol. XXVI, Ed. E. Wolf, Elsevier, Amsterdam 1986.

[3] R.W. Boyd, Nonlinear Optics, Academic Press, London 1992.

[4] W. Jęda, J. Petykiewicz, A. Zagórski, Opt. Appl., in press (1997).

[5] Y.R. Shen, The Principles of Nonlincar Optics, John Wiley \& Sons, New York 1984.

[6] D.A.B. Miller, et al, IEEE J. Quantum Electron. QE-17, 697 (1981).

[7] O. Hess, R. Macdonald, H.J. Eichler, Opt. Commun. 8, 526 (1991).

[8] Ch. Shuchao, II. Rongzhang, Z. IIuiguang, SPIE Proc. 1230, 756 (1990).

[9] N.V. Tabiryan, A.V. Sukhov, B.Ya. Zeldovich, Mol. Cryst. Liq. Cryst. 138, 1 (1986).

[10] I.C. Khoo, S.T. Wu, Optics and Nonlinear Optics of Liquid Crystals, World Scientific, Singapore 1993. 